\title{
Seroprevalencia de citomegalovirus en donantes de órganos y receptores de trasplante renal, Colombia, 2010-2014
}

\author{
Yazmín Rocío Arias-Murillo', Karime Osorio-Arango¹, Jorge Alberto Cortés², Mauricio Beltrán ${ }^{3}$ \\ 1 Subdirección Nacional de Trasplantes y Bancos de Sangre, Dirección de Redes en Salud Pública, Instituto \\ Nacional de Salud, Bogotá, D.C., Colombia \\ 2 Grupo de Investigación en Enfermedades Infecciosas, Departamento de Medicina Interna, Facultad de \\ Medicina, Universidad Nacional de Colombia, Bogotá, D.C., Colombia \\ 3 Dirección Redes en Salud Pública, Instituto Nacional de Salud, Bogotá, D.C., Colombia
}

Introducción. La infección por citomegalovirus ha cobrado gran importancia en los receptores de trasplantes debido a las implicaciones clínicas que puede tener en pacientes inmunocomprometidos.

Objetivo. Describir la seroprevalencia del citomegalovirus en donantes de órganos y receptores de trasplante renal a nivel nacional seleccionados de las seis regionales en que está dividido el país según las áreas de actuación de la Red Nacional de Donación y Trasplante.

Materiales y métodos. Se hizo un estudio descriptivo y retrospectivo que incluyó 1.813 donantes de órganos y 3.313 personas receptoras de trasplante renal, y se calculó la seroprevalencia general de $\lg M$ e IgG para citomegalovirus. La prevalencia de IgG se estratificó por sexo, grupos de edad y regional, se analizó el resultado en cada pareja de donante y receptor, y se estratificó el riesgo. Se utilizaron los paquetes estadísticos IBM SPSS ${ }^{\oplus}$, Statistics 22, y Epi-Info 7.

Resultados. La prevalencia de IgG para citomegalovirus fue de $86,2 \%$ en donantes y de $91,0 \%$ en receptores de trasplante renal, con diferencias estadísticamente significativas por edad, por criterio geográfico y según su calidad de donantes o receptores. Se analizaron 1.764 parejas de donante y receptor, de las cuales $91,4 \%$ se clasificó como de riesgo intermedio.

Conclusiones. Los resultados del presente estudio evidenciaron que las tasas de infección por citomegalovirus fueron altas y que la categorización del riesgo de los receptores de trasplante señala la necesidad de que los equipos médicos tratantes tomen medidas para minimizar los riesgos.

Palabras clave: citomegalovirus, prevalencia, estudios seroepidemiológicos, serología, donantes de tejido, trasplante de riñón.

doi: http://dx.doi.org/10.7705/biomedica.v36i0.2938

Cytomegalovirus seroprevalence in organ donors and kidney transplant recipients, Colombia, 2010-2014

Introduction: Cytomegalovirus infections have gained high importance for individuals that have received organ transplants given the clinical implications this may have in immunocompromised patients.

Objective: To describe the seroprevalence of cytomegalovirus in organ donors and recipients of kidney transplants nationwide from the six regions established by the Red Nacional de Donación y Trasplante.

Materials and methods: We conducted a descriptive retrospective study that included 1,813 organ donors and 3,313 recipients of kidney transplants, and we calculated IgM and IgG seroprevalence for cytomegalovirus. IgG prevalence was stratified according to sex, age group, and region, and the results were analyzed in each donor-recipient pair and classified according to the risk. Statistical packages IBM SPSS ${ }^{\circledR}$, Statistics 22, and Epi Info 7 were utilized.

Results: IgG prevalence for cytomegalivirus was $86.8 \%$ in donors and $91.0 \%$ in kidney transplant recipients with statistical significance observed for age, geographical location, and between donors and recipients. We analyzed 1,764 pairs of donors and recipients, of which $91.4 \%$ were categorized as having intermediate risk.

\footnotetext{
Contribución de los autores:

Yazmín Rocío Arias-Murillo: consolidación y validación de la información, discusión de resultados

Karime Osorio-Arango: consolidación, validación y análisis epidemiológico de los datos

Jorge Alberto Cortés y Mauricio Beltrán: discusión de resultados

Todos los autores participaron en la escritura del manuscrito.
} 
Conclusions: The results of this study showed high cytomegalovirus infection rates in Colombia. Given the risk, categorization of patients undergoing transplants, measures should be adopted by medical teams to minimize risks.

Key words: cytomegalovirus, prevalence, seroepidemiologic studies, serology, tissue donor, kidney transplantation.

doi: http://dx.doi.org/10.7705/biomedica.v36i0.2938

El citomegalovirus pertenece a la familia Herpesviridae y es un virus ampliamente distribuido entre mamíferos; es de reacción específica para la especie y tiene un efecto citopático caracterizado por el aumento del tamaño celular con presencia de inclusiones citoplásmicas e intranucleares. Estos virus comparten la capacidad de permanecer latentes durante largos periodos: después de la infección primaria, entran en los monocitos y en las células precursoras hematopoyéticas y permanecen latentes, generalmente por el resto de su vida, al igual que los anticuerpos de tipo lgG (1).

Es posible que el citomegalovirus se reactive como una nueva infección o como infección recurrente, fenómeno que puede ocurrir en cualquier momento y suele ser más frecuente cuando existe un compromiso inmunitario provocado por otra enfermedad, como el sida o los carcinomas, o por el tratamiento con inmunosupresores con agentes quimioterapéuticos, o por el trasplante de órganos.

En el caso del trasplante renal, el virus puede ser agente causal de infección o de una enfermedad posterior al trasplante, ya sea por reactivación del propio virus latente o por introducción de un virus presente en el órgano del donante. Se le considera un agente patógeno importante en estos pacientes inmunocomprometidos y representa un riesgo que puede estar asociado con la pérdida o la alteración de la función del injerto o con la mortalidad del receptor del trasplante (2-4).

La presencia de anticuerpos en una población indica infecciones previas y varía entre países en desarrollo y países desarrollados, con tasas generalmente bajas en Norteamérica, Europa, y Australia, y tasas de seroprevalencia más altas en África y Asia, las cuales pueden llegar a $100 \%$ en algunos países (5-9).

\section{Correspondencia:}

Yazmín Rocío Arias-Murillo, Subdirección Nacional de

Trasplantes y Bancos de Sangre, Dirección de Redes en Salud

Pública, Instituto Nacional de Salud, Avenida calle 26 N51-20,

Bogotá, D.C., Colombia

Teléfono: (0571) 2200925

ariasmurillo@hotmail.com

Recibido: 14/07/15; aceptado: 03/05/16
El objetivo del presente estudio fue describir la prevalencia de anticuerpos para citomegalovirus en donantes de órganos y receptores de trasplante renal en Colombia.

\section{Materiales y métodos}

Se hizo un estudio descriptivo y retrospectivo sobre la seroprevalencia de la infección por citomegalovirus en donantes de órganos y receptores de trasplante renal. La fuente de información fueron los resultados de las pruebas de laboratorio (anticuerpos de tipo $\lg G$ e $\lg M$ ) reportados al sistema nacional de información de la Red Nacional de Donación y Trasplante durante el periodo de 2010 a 2014 por las instituciones prestadoras de servicios de salud con programas habilitados de trasplante renal.

En el caso de los donantes, el reporte de anticuerpos IgG anti-citomegalovirus se hace en cumplimiento de lo establecido en la normatividad vigente, y en el caso de los receptores corresponde a los resultados reportados como producto del estudio previo al trasplante al que deben someterse los individuos que ingresan en la lista de espera para trasplante renal $(10,11)$.

Las variables sociodemográficas incluidas en la caracterización correspondieron a la edad, el sexo, la regional emisora del reporte y el régimen de afiliación al Sistema General de Seguridad Social en Salud; las variables clínicas incluyeron el grupo sanguíneo y los resultados de las pruebas de anticuerpos IgM e lgG para citomegalovirus.

El análisis se efectuó en tres etapas con los paquetes estadísticos IBM SPSS, Statistics $22^{\circledR}$, y Epi-Info 7, con licencia otorgada al Instituto Nacional de Salud.

Durante la etapa del análisis univariado, las variables cuantitativas se describieron mediante el cálculo de medidas de tendencia central y dispersión, y las cualitativas, mediante frecuencias absolutas y relativas. En la siguiente etapa se calculó la seroprevalencia general de $\operatorname{lgM}$ e IgG para citomegalovirus en donantes de órganos y receptores de trasplante renal, así 
como la prevalencia de IgG para citomegalovirus estratificada por sexo, por grupos de edad y por regional; asimismo, se evaluó la significación de las diferencias de prevalencia entre los grupos estudiados mediante la prueba de ji al cuadrado, para lo cual se consideró como significativo un valor de $p$ menor de 0,05 . Por último, se analizó el resultado de IgG para citomegalovirus de cada pareja de donante y receptor, con el fin de estratificar el riesgo, y se calculó la prevalencia para cada grupo.

\section{Consideraciones éticas}

Este es un estudio retrospectivo que utilizó información secundaria y no presentó ningún riesgo adicional; se utilizaron los datos de donantes y receptores ingresados en el sistema de información de la Red Nacional de Donación y Trasplante en cumplimiento de la normatividad vigente. Las muestras ya se habían recolectado y procesado antes del estudio. No se hizo ninguna intervención o modificación intencionada de variables biológicas, fisiológicas, sicológicas o sociales de los individuos participantes, por lo tanto, el estudio se catalogó como una investigación sin riesgo según la clasificación establecida en la Resolución 8430 de 1993 del Ministerio de Salud. Se protegió la confidencialidad de la información según los lineamientos establecidos y la normatividad de la Red Nacional de Donación y Trasplante (10).

\section{Resultados}

\section{Donantes}

Se analizaron los datos de 1.813 donantes de órganos, de los cuales $70,0 \%$ correspondió a hombres; la mediana de edad fue de 33 años, con un mínimo de un año y un máximo de 69. El mayor porcentaje de donantes se presentó durante el $2010(23,4 \%), 42,0 \%$ de ellos en la regional número 2, la cual corresponde a Medellín; el 54,0 $\%$ se encontraba afiliado al régimen contributivo y $60,0 \%$ pertenecía al grupo sanguíneo O (cuadro 1). Del total de donantes, $59,3 \%$ fue de tipo totipotencial, 33,5\%, multiorgánico, y 7,2 \%, de un solo órgano.

La prevalencia general de IgM para citomegalovirus en los donantes fue de 1,3\% y de 86,2\% para lgG. Al comparar las prevalencias de IgG para el virus no se encontraron diferencias significativas por sexo ni por régimen de afiliación, pero sí entre los menores de 18 y los mayores de 50 años en cuanto a la prevalencia, y entre la regional de Bucaramanga y las de Bogotá y Medellín (cuadro 2).

\section{Trasplante renal}

Se estudiaron 3.313 pacientes con trasplante renal, de los cuales $60,0 \%$ correspondía a hombres, con una mediana de edad de 42,5 años (mínimo de un año y máximo de 76 años). El mayor porcentaje se presentó durante el 2010 (25,8\%); $32,1 \%$ pertenecía a la regional 2 , correspondiente a Medellín; 75,6 \% se encontraba afiliado al régimen contributivo, y 55,5\% pertenecía al grupo sanguíneo $O$, Rh positivo (cuadro 1); 79,0 \% era de raza mestiza y en $93,7 \%$ de los casos el donante había fallecido.

La prevalencia de IgM para citomegalovirus durante el periodo de estudio fue de 3,3\% y, para lgG, de $91,0 \%$. Al comparar la prevalencia de $\operatorname{lgG}$ se encontraron diferencias significativas por sexo, así como entre los menores de 18 y los mayores de 30 años, y entre los pertenecientes a la regional de Bucaramanga y la de Cali; no se encontraron diferencias significativas en cuanto al régimen de afiliación a la seguridad social (cuadro 2).

Cuadro 1. Características generales de donantes y receptores de trasplante renal, Colombia, 2010-2014

\begin{tabular}{|c|c|c|c|c|}
\hline \multirow[t]{2}{*}{ Características } & \multicolumn{2}{|c|}{ Donantes } & \multicolumn{2}{|c|}{$\begin{array}{l}\text { Receptores de } \\
\text { trasplante renal }\end{array}$} \\
\hline & $\mathbf{n}$ & $\%$ & $\mathbf{n}$ & $\%$ \\
\hline \multicolumn{5}{|l|}{ Año } \\
\hline 2010 & 425 & 23,4 & 856 & 25,8 \\
\hline 2011 & 372 & 20,5 & 783 & 23,6 \\
\hline 2012 & 391 & 21,6 & 773 & 23,3 \\
\hline 2013 & 306 & 16,9 & 491 & 14,8 \\
\hline 2014 & 319 & 17,6 & 410 & 12,4 \\
\hline \multicolumn{5}{|l|}{ Sexo } \\
\hline Femenino & 544 & 30 & 1.315 & 40 \\
\hline Masculino & 1.269 & 70 & 1.998 & 60 \\
\hline \multicolumn{5}{|c|}{ Grupos de edad (años) } \\
\hline$<18$ & 193 & 10,7 & 183 & 7,6 \\
\hline $18-29$ & 586 & 32,4 & 400 & 16,7 \\
\hline $30-49$ & 609 & 33,7 & 984 & 41,1 \\
\hline$>50$ & 418 & 23,1 & 829 & 34,6 \\
\hline \multicolumn{5}{|l|}{ Regional } \\
\hline Bogotá & 532 & 29,3 & 1.023 & 30,9 \\
\hline Medellín & 760 & 41,9 & 1.061 & 32,1 \\
\hline Cali & 343 & 18,9 & 791 & 23,9 \\
\hline Bucaramanga & 101 & 5,6 & 234 & 7,1 \\
\hline Barranquilla & 5 & 0,3 & 80 & 2,4 \\
\hline Neiva & 72 & 4 & 120 & 3,6 \\
\hline \multicolumn{5}{|l|}{ Régimen } \\
\hline Contributivo & 879 & 54 & 2.503 & 75,6 \\
\hline Subsidiado & 447 & 27,3 & 804 & 24,3 \\
\hline Otros & 302 & 18,6 & 5 & 0,1 \\
\hline \multicolumn{5}{|l|}{ Grupo sanguíneo } \\
\hline $\mathrm{O}$ & 406 & 64,96 & 526 & 58,38 \\
\hline$A$ & 167 & 26,72 & 279 & 30,97 \\
\hline$B$ & 44 & 7,04 & 77 & 8,55 \\
\hline$A B$ & 8 & 1,28 & 19 & 2,11 \\
\hline
\end{tabular}


Cuadro 2. Prevalencia de IgG para citomegalovirus en donantes y receptores de trasplante renal, Colombia, 2010-2014

\begin{tabular}{|c|c|c|c|c|c|c|c|c|}
\hline \multirow{2}{*}{ Factor } & \multicolumn{4}{|c|}{ Donantes } & \multicolumn{4}{|c|}{ Trasplantados renales } \\
\hline & Positivo (\%) & Negativo (\%) & & p & Positi & vo (\%) & Negativo (\%) & $\mathbf{p}$ \\
\hline \multicolumn{9}{|l|}{ Sexo } \\
\hline Femenino & $472(86,8)$ & $72(13,2)$ & \multirow{2}{*}{0,24} & \multirow{2}{*}{$(0,62)$} & 1.214 & $(92,3)$ & $101(7,7)$ & \multirow{2}{*}{$4,40(0,03)$} \\
\hline Masculino & $1.090(85,9)$ & $179(14,1)$ & & & 1.802 & $(90,2)$ & $196(9,8)$ & \\
\hline \multicolumn{9}{|c|}{ Grupos de edad (años) } \\
\hline$<18$ & $157(81,3)$ & $36(18,7)$ & & 1 & 155 & $(84,7)$ & $28(15,3)$ & 1 \\
\hline $18-29$ & $509(86,9)$ & $77(13,1)$ & 3,55 & $(0,05)$ & 351 & $(87,7)$ & $49(12,3)$ & $1,01(0,31)$ \\
\hline $30-49$ & $525(86,2)$ & $84(13,8)$ & 2,72 & $(0,09)$ & 890 & $(90,4)$ & $94(10,6)$ & $5,44(0,01)$ \\
\hline$>50$ & $366(87,6)$ & $52(12,4)$ & 4,13 & $(0,04)$ & 761 & $(91,8)$ & $68(8,2)$ & $8,79(0,00)$ \\
\hline \multicolumn{9}{|l|}{ Regional } \\
\hline Bogotá & $462(86,8)$ & $70(13,2)$ & 5,08 & $(0,02)$ & 930 & $(90,9)$ & $93(9,1)$ & $3,00(0,08)$ \\
\hline Medellín & $668(87,9)$ & $92(12,1)$ & 7,26 & $(0,00)$ & 956 & $(90,1)$ & $105(9,9)$ & $1,75(0,18)$ \\
\hline Cali & $292(85,1)$ & $51(14,9)$ & 2,71 & $(80,09)$ & 742 & $(93,8)$ & $49(6,2)$ & $11,14(0,00)$ \\
\hline Bucaramanga & $79(78,2)$ & $22(21,8)$ & 1 & & 204 & $(87,2)$ & $30(12,8)$ & 1 \\
\hline Barranquilla & $4(80,0)$ & $1(20,0)$ & 0,00 & $(0,92)$ & 70 & $(87,5)$ & $10(12,5)$ & $0,00(0,94)$ \\
\hline Neiva & $57(79,2)$ & $15(20,8)$ & 0,02 & $(0,88)$ & 110 & $(91,7)$ & $10(8,3)$ & $1,59(0,20)$ \\
\hline \multicolumn{9}{|l|}{ Régimen } \\
\hline Contributivo & $751(85,4)$ & $128(14,6)$ & 0,00 & $(0,96)$ & 2.270 & $(90,7)$ & $233 \quad(9,3)$ & $3,57(0,05)$ \\
\hline Subsidiado & $392(87,7)$ & $55(12,3)$ & 0,83 & $(0,36)$ & 470 & $(88,0)$ & $64(12,0)$ & 1 \\
\hline Otro & $257(85,1)$ & $45(14,9)$ & 1 & & & (100) & 0 & $0,68(0,40)$ \\
\hline
\end{tabular}

Los números en negrilla indican las diferencias estadísticamente significativas.

Se comparó la prevalencia de $\lg G$ entre donantes (91\%) y receptores de trasplante renal $(86,2 \%)$, y se encontraron diferencias estadísticamente significativas entre los grupos.

Se analizaron 1.764 parejas de donante y receptor y de su respectivo estado serológico, y se encontró que $91,4 \%$ de los receptores de trasplante de riñón se clasificaron como de riesgo intermedio y $7,3 \%$ como de riesgo alto (cuadro 3 ).

\section{Discusión}

En este estudio se confirmó la alta prevalencia de la infección por citomegalovirus en donantes y receptores de trasplante renal en Colombia. Aunque se registraron diferencias entre los donantes y los receptores de trasplante, los grupos de edad y las regionales, estas fueron relativamente pequeñas $y$, en su conjunto, muestran que Colombia aún tiene tasas de infección por citomegalovirus equiparables a las de otros países en desarrollo (6). La prevalencia de citomegalovirus difiere entre regiones geográficas y está relacionada con el nivel de desarrollo de los países: la seroprevalencia es baja en países de Norteamérica, Europa y Australia, con tasas de prevalencia de $40 \%$, y alcanza tasas de $100 \%$ en países de Asia y África (5-8).

En general, las características sociodemográficas de los donantes de este estudio fueron similares a las reportadas por la Red Nacional de Donación y Trasplante en sus informes anuales, en los cuales
Cuadro 3. Estado serológico y estratificación del riesgo de donantes (D) y receptores (R) de trasplante renal, Colombia, 2010-2014

\begin{tabular}{lcrr}
\hline $\begin{array}{l}\text { Estratificación } \\
\text { del riesgo }\end{array}$ & $\begin{array}{c}\text { Estado } \\
\text { serológico }\end{array}$ & $\mathbf{n}$ & $\%$ \\
\hline Alto & D+/R- & 128 & 7,3 \\
Intermedio & D-/R+ & 180 & 10,2 \\
& D+/R+ & 1.433 & 81,2 \\
Bajo & D-/R- & 23 & 1,3 \\
\hline
\end{tabular}

el mayor porcentaje de donantes corresponde a hombres jóvenes, lo cual se relaciona directamente con las condiciones sociales y las causas de muerte de los donantes de órganos en el país (12-15).

El mayor número de donantes y receptores de trasplante renal se presentó en el 2010, año durante el cual la Red de Donación y Trasplante presentó su máxima actividad, con una tasa de donación de 10 donantes por cada millón de habitantes. La mayoría de donantes pertenecía al grupo sanguíneo $O$, lo cual obedece a que se trata del tipo predominante entre los donantes potenciales, los receptores en lista de espera y la población colombiana en general (14-16).

En algunos estudios llevados a cabo en Serbia, Alemania y República Checa se encontraron prevalencias de IgG para citomegalovirus de 99,0, 83,0 y $80,0 \%$, respectivamente, en pacientes sometidos a procesos de hemodiálisis (17-19). En un estudio 
de la República de Croacia, se evidenció que 90,7 $\%$ de los pacientes sometidos a hemodiálisis había sido positivo para IgG de citomegalovirus, mientras que en la población de control este porcentaje fue de 81,9 , con diferencias significativas que sugirieron que la hemodiálisis constituía un factor de riesgo para dicha positividad (20).

Los resultados en los donantes de este estudio indicaron que la prevalencia fue de $86,2 \%$, con diferencias entre zonas geográficas; los análisis correspondieron a la división por regionales que opera en Colombia para las actividades de donación y trasplante, según lo establecido en las resoluciones 2640 de 2005 y 3272 de 2011 del Ministerio de la Protección Social. Teniendo en cuenta este criterio geográfico, se encontró una menor seroprevalencia de IgG para citomegalovirus en la regional 4 (Bucaramanga), lo cual podría estar asociado a condiciones socioeconómicas; sin embargo, es necesario indicar que el número de individuos de esta regional es mucho menor que el de las regionales 2 (Medellín) y 1 (Bogotá), las cuales tuvieron la mayor representación entre la población estudiada, así como las cifras más altas de seroprevalencia.

Se determinó la edad como una variable asociada a una mayor prevalencia de anticuerpos IgG para citomegalovirus, tanto en donantes de órganos como en los receptores de trasplante renal, con una diferencia significativa entre menores de 18 años y mayores de 50, lo cual indica que a medida que aumentaba la edad se incrementaron los resultados positivos para anticuerpos anticitomegalovirus, tal como se ha reportado en estudios en diferentes poblaciones $(8,17-26)$, y que la mayoría de las infecciones ocurren durante la niñez, lo que explica que el riesgo de infección en los adultos de Colombia sea inferior a $10 \%$. La poca frecuencia de infección primaria en adultos es relevante para los pacientes con trasplante, pues estos derivan su riesgo de enfermedad casi exclusivamente de la presencia de infección previa en el individuo receptor o en el donante del órgano. En los donantes y receptores negativos, el riesgo de enfermedad por citomegalovirus es extremadamente bajo.

Una de las implicaciones de este estudio se relaciona con el riesgo de infección o su reactivación en los pacientes receptores del trasplante. El riesgo de infección o de enfermedad por citomegalovirus en receptores de trasplante está determinado por diversas características del huésped, entre las cuales cabe mencionar las enfermedades concomitantes, la edad, los factores genéticos y los factores de alto impacto, como la inmunosupresión y el estado serológico del citomegalovirus determinado por la asociación entre la presencia de $\lg G$ en el donante y en el receptor, lo cual permite establecer categorías de riesgo alto, intermedio y bajo $(4,27)$.

En estudios anteriores en Colombia, Correa, et al., reportaron en 1989 tasas de prevalencia más bajas en donantes $(81 \%)$ y en receptores ( $89 \%)$, con mayor prevalencia en los receptores de trasplante renal; de igual forma, reportaron $76 \%$ de parejas positivas de donante y receptor (28). Los resultados del presente estudio evidenciaron porcentajes más altos $(81,2 \%)$, con una muestra mucho más representativa de individuos de diferentes zonas geográficas del país.

En la población estudiada, $91,4 \%$ de los pacientes con trasplante de riñón tenían riesgo intermedio, mientras que $7,3 \%$ eran de riesgo alto. Estos datos tienen repercusiones en la planeación de las estrategias de prevención de la reactivación, la infección o el desarrollo de la enfermedad entre los pacientes sometidos a trasplantes. Un estudio previo en Medellín encontró que la frecuencia de pacientes de bajo riesgo era de $0,8 \%$, de riesgo intermedio, 90,9 \% y de alto riesgo, 8,2 \% (29). Estos datos sugieren que la mayoría de los pacientes requieren una estrategia preventiva (profilaxis o una estrategia anticipativa), pues menos de $10 \%$ se consideraría de bajo riesgo y no requeriría de ninguna estrategia preventiva $(30,31)$. En los estudios de cinética viral se ha validado esta aproximación, y se ha demostrado que las tasas de replicación se acercan a $0 \%$ en los grupos de bajo riesgo, fluctúan entre 40 y $60 \%$ en los grupos de riesgo intermedio y son cercanas a $90 \%$ en los de alto riesgo.

Una limitación importante de este estudio fue su carácter retrospectivo, ya que no se pudo determinar la conducta adoptada en el seguimiento de los individuos sometidos a trasplante. Sin embargo, considerando el comportamiento del citomegalovirus y la prevalencia determinada en la población, el panorama que se evidenció refleja el riesgo de infección o enfermedad en los pacientes con trasplante renal en Colombia y plantea la necesidad de implementar estrategias de prevención en el manejo de las personas sometidas a trasplantes para minimizar los riesgos de infección y de enfermedad por citomegalovirus. 


\section{Agradecimientos}

Al Instituto Nacional de Salud, especialmente a la Dirección de Redes en Salud Pública, la Subdirección Nacional de Trasplantes y Bancos de Sangre, la Coordinación Nacional de Donación y Trasplantes, y a la Oficina de Tecnologías de la Información y la Comunicación.

\section{Conflicto de intereses}

Los autores declaran que no tienen ningún conflicto de intereses con respecto a los resultados del presente estudio.

\section{Financiación}

Este estudio fue financiado por el Instituto Nacional de Salud, Dirección de Redes en Salud Pública.

\section{Referencias}

1. Hansen SG, Powers CJ, Richards R, Ventura AB, Ford JC, Siess D, et al. Evasion of CD8+ $T$ cells is critical for superinfection by cytomegalovirus. Science. 2010;328:102-6. http://dx.doi.org/10.1126/science.1185350

2. Schnitzler MA, Lowell JA, Hardinger KL, Boxerman SB, Bailey TC, Brennan DC. The association of cytomegalovirus sero-pairing with outcomes and costs following cadaveric renal transplantation prior to the introduction of oral ganciclovir CMV prophylaxis. Am J Transplant. 2003;3:445-51. http://dx.doi.org/10.1034/j.1600-6143.2003. 00069.x

3. Arthurs SK, Eid AJ, Pedersen RA, Kremers WK, Cosio FG, Patel R, et al. Delayed-onset primary cytomegalovirus disease and the risk of allograft failure and mortality after kidney transplantation. Clin Infect Dis. 2008;46:840-6. http:// dx.doi.org/10.1086/528718

4. Bataille S, Moal V, Gaudart J, Indreies M, Purgus R, Dussol B, et al. Cytomegalovirus risk factors in renal transplantation with modern immunosuppression. Transpl Infect Dis. 2010;12:480-8. http://dx.doi.org/10.1111/j.13993062.2010.00533.x

5. Enders G, Daiminger A, Lindemann L, Knotek F, Bäder $\mathrm{U}$, Exler S, et al. Cytomegalovirus (CMV) seroprevalence in pregnant women, bone marrow donors and adolescents in Germany, 1996-2010. Med Microbiol Immunol. 2012;201: 303-9. http://dx.doi.org/10.1007/s00430-012-0232-7

6. Cannon MJ, Schmid DS, Hyde TB. Review of cytomegalovirus seroprevalence and demographic characteristics associated with infection. Rev Med Virol. 2010;20:202-13. http://dx.doi.org/10.1002/rmv.655

7. Lopo S, Vinagre E, Palminha P, Paixao MT, Nogueira P, Freitas MG. Seroprevalence to cytomegalovirus in the Portuguese population, 2002-2003. Euro Surveill. 2011;16:19896.

8. Vilibic-Cavlek T, Ljubin-Sternak S, Ban M, Kolaric B, Sviben M, Mlinaric-Galinovic G. Seroprevalence of TORCH infections in women of childbearing age in Croatia. J Matern Fetal Neonatal Med. 2011;24:280-3. http://dx.doi. org/10.3109/14767058.2010.485233.
9. Adjei AA, Armah HB, Gbagbo F, Boamah I, Adu-Gyamfi C, Asare I. Seroprevalence of HHV-8, CMV and EBV among the general population in Ghana, West Africa. BMC Infect Dis. 2008;8:111. http://dx.doi.org/10.1186/1471-2334-8-111

10. Ministerio de Salud y Protección Social. Decreto 2493 de 2004. Por el cual se reglamentan parcialmente las Leyes 9a de 1979 y 73 de 1988, en relación con los componentes anatómicos. Diario Oficial No.45631. Bogotá: Imprenta Nacional de Colombia; 2004.

11. Instituto Nacional de Salud. Documento técnico nacional.

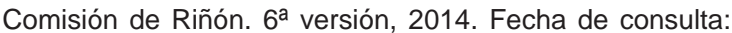
10 de junio de 2015. Disponible en: http://www.ins.gov.co/ lineas-de-accion/Red-Nacional-Laboratorios/Marco\%20 Legal\%20documentos\%20tcnicos\%20y\%20estadistica\%20 red\%20d/COMISION\%20RI\%C3\%91ON\%20Version\%20 No\%206\%20-2014.pdf.

12. Instituto Nacional de Salud. Informe red donación y trasplantes 2010. Fecha de consulta: mayo 10 del 2015. Disponible en: http://www.ins.gov.co/lineas-de-accion/RedNacional-Laboratorios/Estadsticas/Informe\%20FinalRed\%20 D\%20Tx\%20-\%202010.pdf.

13. Instituto Nacional de Salud. Informe anual, Red de Donación y Trasplantes. Volumen 1, Bogotá, 2011. Fecha de consulta: 10 de mayo de 2015. Disponible en: http://www. ins.gov.co/lineas-de-accion/Red-Nacional-Laboratorios/ Estadsticas/Informe\%20Red\%20Donacion\%20y\%20 Trasplantes\%20\%20Vol\%201\%202011.pdf.

14. Instituto Nacional de Salud. Informe anual, Red de Donación y Trasplantes. Volumen 2, Bogotá, 2012. Fecha de consulta: 10 de mayo de 2015. Disponible en: http://www. ins.gov.co/lineas-de-accion/Red-Nacional-Laboratorios/ Estadsticas/Informe_Red\%20donacion $\% 20$ y $\% 20$ trasplantes\%20Vol2_2012.pdf.

15. Instituto Nacional de Salud. Informe anual, Red de Donación y Trasplantes. Volumen 3, Bogotá, 2013. Fecha de consulta: 10 de mayo de 2015. Disponible en: http://www. ins.gov.co/lineas-de-accion/Red-Nacional-Laboratorios/ Estadsticas/INFORME\%20ANUAL\%202013\%20RED\%20 DE\%20DONACION\%20Y\%20TRASPLANTES.\%20 Vol\%2003.pdf.

16. Beltrán M, Ayala $\mathbf{M}$, Jara J. Frecuencia de grupos sanguíneos y factor Rh en donantes de sangre, Colombia, 1996. Biomédica. 1999;19:39-44. http://dx.doi.org/10.7705/ biomedica.v19i1.1006

17. Korcáková L, Kaslík J, Svobodová J, Kaslíková J, Bláha $\mathrm{J}$, Sedlácková E, et al. CMV infection in patients with chronic renal failure and in those following transplantation. Czech Med. 1988;11:131-6.

18. Sibrowski W, Kühnl P, Kalmar G, Albert S, Böhm BO, Doerr HW. Cytomegalovirus diagnosis in blood donors and risk patients. Beitr Infusionsther. 1990;26:37-9.

19. Trkulic M, Jovanovic D, Ostojic G, Kovacevic Z, Taseski J. Cytomegalovirus infection in patients with kidney diseases. Vojnosanit Pregl. 2000;57:63-7.

20. Vilibic T, Kolaric B, Ljubin S, Kos M, Kaic B, Mlinaric G. Prevalence and dynamics of cytomegalovirus infection among patients undergoing chronic hemodialysis. Indian J Nephrol. 2015;25:95-98. http://dx.doi.org/10.4103/09714065.139488 
21. Betjes MG, Litjens NH, Zietse R. Seropositivity for cytomegalovirus in patients with end-stage renal disease is strongly associated with atherosclerotic disease. Nephrol Dial Transplant. 2007;22:3298-303. http://dx.doi. org/10.1093/ndt/gfm348

22. Kao TW, Hsu WA, Chen HS, Chen WY. A two year followup study of common virus infections in hemodialysis patients in Taiwan. Artif Organs. 2002;26:879-83. http://dx. doi.org/10.1046/j.1525-1594.2002.07053.x

23. Jha V. Post-transplant infections: An ounce of prevention. Indian J Nephrol. 2010;20:171-8. http://dx.doi.org/10 4103/0971-4065.73431

24. Cordero E, Casasola C, Ecarma R, Danguilan R. Cytomegalovirus disease in kidney transplant recipients: Incidence, clinical profile, and risk factors. Transplant Proc. 2012;44:694-700. http://dx.doi.org/10.1016/j.transproceed. 2011.11.053

25. Cavdar C, Celtik A, Saglam F, Sifil A, Atila K, Celik A, et al. Cytomegalovirus disease in renal transplant recipients: A single-center experience. Ren Fail. 2008;30:503-6. http:// dx.doi.org/10.1080/08860220802064705

26. Enan KA, El-Hussein AM, El Eragi A, Elkhider IM. Seroreactivity to human cytomegalovirus in a cohort of Sudanese renal transplant patients. Khartoum Med J. 2009;2:197-9.
27. Sagedal S, Nordal KP, Hartmann A, Degre M, Holter E, Foss A, et al. A prospective study of the natural course of cytomegalovirus infection and disease in renal allograft recipients. Transplantation. 2000;70:1166-74.

28. Correa MR, Correa M, Arbeláez M, Builes M, Ossa J. Prevalencia de infección por citomegalovirus en receptores y donantes de trasplante renal en Medellín para 1988-1989. Acta Médica Colombiana. 1990;15:175-9.

29. Diaz J, Henao J, Rodelo J, García A, Arbeláez M, Jaimes F. Incidence and risk factors for cytomegalovirus disease in a Colombian cohort of kidney transplant recipients. Transplant Proc. 2014;46:160-6. http://dx.doi.org/10.1016/j. transproceed.2013.07.070

30. Kotton CN, Kumar D, Caliendo AM, Asberg A, Chou S, Danziger-lsakov L, et al. Updated international consensus guidelines on the management of cytomegalovirus in solidorgan transplantation. Transplantation. 2013;96:333-60. http://dx.doi.org/10.1097/TP.0b013e31829df29d

31. Cortés JA, Yomayusa N, Arias YR, Arroyave IH, Cataño JC, García P, et al. Consenso colombiano para la estratificación, diagnóstico, tratamiento y prevención de la infección por citomegalovirus en pacientes adultos con trasplante renal. Infectio. 2016. http://dx.doi.org/10.1016/j. infect.2015.10.005 